\title{
$\operatorname{micr} \oplus$ biology $\gg \mapsto \gg$
}

$\mapsto \mapsto !$...Comment ...?"

Microbiology Comment provides a platform for readers of Microbiology to communicate their personal observations and opinions in a more informal way than through the submission of papers.

Most of us feel, from time to time, that other authors have not acknowledged the work of our own or other groups or have omitted to interpret important aspects of their own data. Perhaps we have observations that, although not sufficient to merit a full paper, add a further dimension to one published by others. In other instances we may have a useful piece of methodology that we would like to share.

The Editors hope that readers will take full advantage of this section and use it to raise matters that hitherto have been confined to a limited audience.

Jon Saunders, Editor-in-Chief

\section{Stabilization of Rhizobium symbiosis plasmids}

The ability of Rhizobium sp. NGR234 to participate in symbiosis is determined by a $536 \mathrm{~kb}$ symbiotic plasmid, pNGR234a (5). This plasmid contains genes essential for symbiosis, but NGR234 can be cured of pNGR234a, yielding strain ANU265 (10) which, although incapable of symbiosis, is viable. Therefore, to retain such a large plasmid without obvious selection there must exist some system of plasmid stabilization.

Bacteria utilize a number of mechanisms to maintain low-copy-number plasmids (6-8). These can be divided into mechanisms that actively distribute the plasmid to daughter cells at cell division, those which resolve plasmid oligomers (when they occur) into monomers and those which cause the death of plasmid-free daughter cells by postsegregational killing. In all cases of postsegregational killing, a plasmid-encoded gene system produces a stable poison and an unstable antidote. In a plasmid-deficient offspring a protein antidote is more readily digested by cellular proteases than the poison and the cell is killed by the toxic effects of the latter. The chromosomally encoded two-gene hip operon of Escherichia coli described by us (4) and others $(2,3,11,12,14)$ exhibits many of the characteristics of the two-protein plasmid stabilization system, other examples of which include $c c d$ of the $\mathrm{F}$ plasmid, parD/pem of plasmids R1 and R100, parDE of plasmids RP4 and RK2, and phd/doc of the P1 plasmid (for review, see ref. 8). The hip operon, at $33.8 \mathrm{~min}$ on the E. coli chromosome, consists of two genes, $\operatorname{hip} A$ and $h i p B$, encoding proteins of 50 and $10 \mathrm{kDa}$, respectively. Under normal conditions, HipA and HipB cannot be isolated unbound to one another (3). If $h i p A$, encoding the poison, is expressed in excess of $\operatorname{hip} B$, encoding the antidote, then E. coli cell division is rapidly inhibited (4). As in other stabilization systems, hipA and $h i p B$ are adjacent genes in an operon in which the antidote is encoded by the upstream gene of the operon and the bound complex of antidote and poison acts to autoregulate the system by binding an upstream regulatory region (3). In addition to functioning as an antidote, HipB, a Crolike protein, also acts as a repressor reducing the activity of the hip promoter to barely detectable levels (3). This, coupled to the fact that the stop codon of $h i p B$ overlaps the start codon of hipA, indicates the presence of strict transcriptional and translational regulation to keep levels of unbound HipA, the poison, to an absolute minimum. It could therefore be concluded that, if expressed on a plasmid, the hip operon would act as a stabilization system in which plasmid loss would result in HipB being more rapidly degraded than HipA causing death of the plasmid-free cell.

There are examples of chromosomal operons that have homology to plasmidencoded genes responsible for plasmid maintenance, such as $c h p A$ and $c h p B$, which have homology with pem of the R100 plasmid (9). As with hip, the function of such chromosomal systems is unknown, but ChpA and $\mathrm{ChpB}$ have similar effects upon cell growth as the corresponding Pem protein. In the case of $\operatorname{chp} A$ and $\operatorname{chp} B$, homologues do exist on E. coli plasmids. However, in the case of hip there appear to be only two intact homologues of the poison gene hipA, and both are on the R hizobium symbiosis plasmid pNGR234a (5). These are genes y $4 \mathrm{mE}$ and $\mathrm{y} 4 \mathrm{dM}$, previously of unassigned function, but encoding proteins with 28 and $27 \%$ identity, respectively, to HipA. As in the case of bipA and $h i p B$, both $y 4 \mathrm{mE}$ and $\mathrm{y} 4 \mathrm{dM}$ are downstream of repressors $y 4 \mathrm{mF}$ and $\mathrm{y} 4 \mathrm{dL}$, the stop codons of which overlap the start codons of $\mathrm{y} 4 \mathrm{mE}$ and $\mathrm{y} 4 \mathrm{dM}$, respectively, This strongly indicates that, as seen in E. coli, unregulated expression of the hipA analogues would be detrimental to the cell (4). It is therefore tempting to speculate that $\mathrm{y} 4 \mathrm{mE} / \mathrm{mF}$ and $\mathrm{y} 4 \mathrm{dM} / \mathrm{dL}$ act as plasmid stabilization systems for the symbiosis plasmid pNGR234a, a plasmid that can be cured from Rhizobium without affecting survival of the organism (10).

The known targets for proteic killer genes include DNA gyrase $(c c d)$ (1) and DnaB (pem) (13). In the case of hip the target is unlikely to be DNA gyrase as its inhibition leads to filamentation of $E$. coli, a phenomenon not observed with induction of $h i p A$ (4). The parD and pem systems of plasmids R1 and $\mathrm{R} 100$ are located close to their origins of replication and this is also the case for one of the hip homologues ( $44 \mathrm{dM}$ ) in Rhizobium in which the locus is situated equidistant between the origin of replication (oriV) and origin of transfer (oriT). The pem system consists of PemK (poison) and PemI (antidote) and the target of the system is believed to be DnaB, as DnaB-dependent initiation of DNA replication is inhibited by the addition of PemK in vitro (13). Due to the similar location of the hip analogue to

\section{GUIDELINES}

Communications should be in the form of letters and should be brief and to the point. A single small Table or Figure may be included, as may a limited number of references (cited in the text by numbers, and listed in alphabetical order at the end of the letter). A short title (fewer than 50 characters) should be provided.

Approval for publication rests with the Editor-in-Chief, who reserves the right to edit letters and/or to make a brief reply. Other interested persons may also be invited to reply. The Editors of Microbiology do not necessarily agree with the views expressed in Microbiology Comment.

Contributions should be addressed to the Editor-in-Chief via the Editorial Office. 
the origin of replication of the symbiosis plasmid, DnaB is a possible $\mathrm{y} 4 \mathrm{dM}$ target in Rhizobium.

This is the first evidence that a plasmid stabilization system may exist in Rhizobium and suggests a mechanism by which such a system might operate to retain certain symbiosis plasmids.

\section{T. J. Falla and I. Chopra*}

Antimicrobial Research Centre and Department of Microbiology, University of Leeds, Leeds LS2 9JT

*For correspondence. Tel: +441132335604. Fax: +44 1132335638 .

e-mail: micic@leeds.ac.uk

1. Bernard, P. \& Couturier, M. (1992). Cell killing by the $\mathrm{F}$ plasmid $\mathrm{CcdB}$ protein involves poisoning of DNA-topoisomerase II complexes. J Mol Biol 226, $73.5-745$

2. Black, D. S., Kelly, A. J., Mardis, M. J. \& Moyed, H. S. (1991). Structure and organization of bip, an operon that affects lethality due to inhibition of peptidoglycan or DNA synthesis. J Bacteriol 173, $5732-5739$

3. Black, D. S., Irwin, B. \& Moyed, H. S. (1994). Autoregulation of hip, an operon that affects lethality due to inhibition of peptidoglycan or DNA synthesis. J Bacteriol 176, 4081-4091.

4. Falla, T. F. \& Chopra, I. (1998). Joint tolerance to $\beta$-lactam and quinolone antibiotics in Escherichia col is due to over-expression of hipA. Antimicrob Agents Chemother 42, 3282-3284.

5. Freiberg, C., Fellay, R., Bairoch, A., Broughton, W. J., Rosenthal, A. \& Perret, X. (1997). Molecular basis of symbiosis between Rhizobium and legumes. Nature 387 . $394-401$.

6. Gerdes, K., Poulson, L. K., Thisted, T., Nielsen, A. K., Matinussen, J. \& Andreasen, P. H. (1990). The bok killer gene family in gram-negative bacteria. New Biol 2, 946-956.

7. Holcík, M. \& Iyer, V. N. (1997). Conditionally lethal genes associated with bacterial plasmids. Microbiology $143,3403-3416$.

8. Jensen, R. B. \& Gerdes, K. (1995). Programmed cell death in bacteria: proteic plasmid stabilization systems. Mol Microbiol 17, 205-210.

9. Masuda, Y., Miyakawa, K., Nishimura, Y. \& Ohtsubo, E. (1993). chpA and chpB, Escherichia coli chromosomal homologs of the pem locus responsible for the stable maintenance of plasmid R100. J Bacteriol $175,6850-6856$.

10. Morrison, N. A., Cen, Y. H., Chen, H. C., Plazinski, J., Ridge, R. \& Rolfe, B. G. (1984). Mobilization of a sym plasmid from a fast-growing cowpea Rhizobium strain. J Bacteriol 160, 483-487. 11. Moyed, H. S. \& Bertrand, K. P. (1983). hipA, a newly recognized gene of Escherichia coli $\mathrm{K}-12$ that affects frequency of persistence after inhibition of murein synthesis. J Bacteriol 155, 768-775.

12. Moyed, H. S. \& Broderick, S. H. (1986). Molecular cloning and expression of hipA gene of Escherichia coli $\mathrm{K}-12$ that affects frequency of persistence after inhibition of murein synthesis. J Bacteriol 166, 399-403.

13. Ruiz-Echevarría, M. J., Giménez-Gallego, G., Sabariegos-Jareno, R. \& Díaz-Orejas, R. (1995). Kid, a small protein of the parD stability system of plasmid $R 1$, is an inhibitor of DNA replication acting at the initiation of DNA synthesis. Mol Microbiol 247, 568-577.

14. Wolfson, J. S., Hooper, D. C., McHugh, G. L., Bozza, M. A. \& Swartz, M. N. (1990). Mutants of Escherchia coli $\mathrm{K}-12$ exhibiting reduced killing by both quinolone and $\beta$-lactam antimicrobial agents. Antimicrob Agents Chemother 34, 1938-1943.

\section{A complex group I intron in Nectria galligena rDNA}

An article by Crockard and coworkers in a recent issue of Microbiology reported a polymorphic insertion element of 363,1185 or $1423 \mathrm{bp}$ within the nuclear small subunit (SSU) rDNA of the ascomycete fungus Nectria galligena (3). The insertion site was mapped to position 1199 (corresponding to Escherichia coli SSU rRNA numbering), a site known to harbour nuclear group I introns in fungi. However, the authors failed to assign this insertion to any known class of insertion elements.

Several classes of insertions (introns and retroelements) are known to interrupt genes encoding rRNA, with the group I introns being the most frequent (10). Despite the fact that all group I introns perform the same catalytic reaction at the RNA level, resulting in intron excision and exon ligation (1), almost none of the sequence positions are universally conserved. However, the group I intron RNAs can be folded into a common well-defined secondary and threedimensional structure (12), and 12 subgroups, based on distinct primary sequence motifs and characteristic structural features in peripheral regions, have been proposed $(5,13)$.

We have re-analysed the Nectria sequences reported by Crockard et al. (3) and discovered that the smallest insertion (the 363 intron) contains a typical group I intron structure (Fig. 1a). The intron, which belongs to subgroup IC4, is distantly related to the more common subgroup IC1 introns in nuclear rDNA (5). A homologous structure is also present at the $5^{\prime}$ end of the Nectria intermediate-sized insertion (the 1185 intron). We have previously reported functional analyses of a group IC4 intron in the SSU rDNA of the myxomycete Didymium $(4,11)$. The group IC4 intron follow; the same splicing pathway as the prototype group IC1 intron from Tetrahymena (1).

A FASTA search (GCG Computer Package) using the 363 intron sequence as a 'probe' identifies three related fungal group IC4 introns inserted at the SSU rDNA position 1199 (the same as the Nectria intron). These include two Cryphonectra introns (2) and an intron from Tilletiopsis flava (15). The fact that similar group IC4 introns are found in distantly related eukaryotic micro-organisms suggests that the Nectria 363 intron, in contrast to what was suggested by Crockard et al. (3), has been gained by horizontal transfer during evolution.

Some nuclear group I introns are known to contain ORFs encoding functional endonucleases involved in intron mobility by homing at the DNA level $(6,8,14)$. All these nuclear homing endonucleases contain the characteristic His-Cys box motif of a 30 -aa

\section{(a) Group IC4 intron}

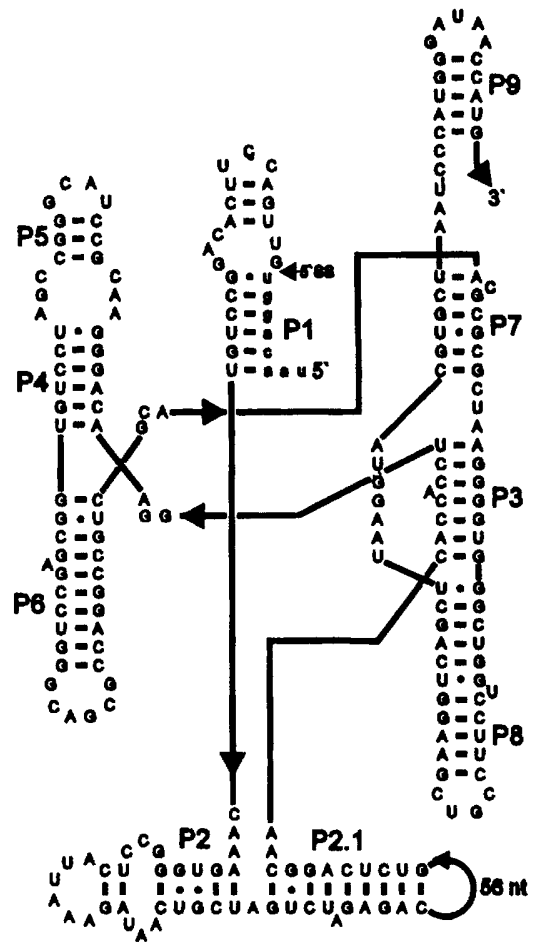

(b)

\section{His-Cys box}

\section{NoC. OHLGGUPL GLEPAHIHVEPKTAMEARKG Phy. OHLGHITRGHNPL HLCWESL DOUKGRNWE Na. OHLGGUGGGARPGHLR I EKKSVIDERT HE Por. BHTCHIAKGVNKAHLT LESGDL IKSR I Y}

Fig. 1. Hallmarks of nuclear group I introns within the rDNA insertions of Nectria galligena. (a) Secondary structure model of the group IC4 intron present in both the 363 and 1185 introns. Paired segments $(\mathbf{P})$ are indicated. The $5^{\prime}$ exon sequence is represented in lower case letters. The sequence of the 363 intron is shown. (b) The HisCys box motif from Nectria galligena (Nec.) 1185 and 1423 introns aligned to the corresponding motifs (9) present in nuclear group I introns from Physarum polycephalum (Phy.), Naegleria jamiesoni (Nae.) and Porphyra spiralis (Por.).

histidine- and cysteine-rich sequence (9). An identical His-Cys box is shared by the 1185 intron and in the large Nectria insertion (the 1423 intron), and the motif in Nectria (Fig. 1b) is very similar to those previously reported in homing endonucleases from the myxomycete Physarum, the amoeboflagellate Naegleria and the red alga Porphyra (8). The Physarum homing endonuclease is well studied and known to be directly involved in group I intron mobility (14). The recently deduced crystal structure of this endonuclease suggests that the His-Cys box residues are directly involved in novel zincbinding motifs and in the active site of the enzyme (7). In Nectria, the His-Cys box- 